\title{
Complex atmospheric-lithospheric observations of acoustic emission at «Karymshina» site in Kamchatka
}

\author{
Igor Larionov ${ }^{1, \star}$ \\ ${ }^{1}$ Institute of Cosmophysical Research and Radio Wave Propagation FEB RAS, Paratunka, Russia
}

\begin{abstract}
The results of complex observations of acoustic emission in the near surface rocks and in the atmosphere by the ground surface are described. The instrumentations for the observations are a laser strainmeter-interferometer and a microbarometer installed close to each other. It was shown that during the increase of deformation rate in the near surface rocks, increase of acoustic emission intensity in the atmosphere by the ground surface is registered. The effect of meteorological factors on the observation results is evaluated.
\end{abstract}

\section{Introduction}

At present time, the topical task in Acoustics is the joint investigation of acoustic wave processes at the lithosphere and atmosphere boundary. Investigation of acoustic waves, propagating in "solid body - gas" system, is necessary to built the models of lithosphere, atmosphere, ionosphere and magnetosphere coupling which is one of the main directions in Geophysics.

The lithospheric-atmospheric effect manifests the most in seismically active regions at the filal stage of earthquake preparation [1]. The high interest to the joint research of seismically active wave processes is determined by the fact that acoustic waves of infrasonic range can propagate at large distances and reach ionospheric heights under favorable conditions (weak attenuation and scattering, weak refraction in the atmosphere) [2]. As a result, they play a significant role in energy transfer between the geospheres.

\section{Measurement technique}

In 2016, a laser strainmeter-interferometer, constructed according to Maikelson interferometer scheme, was put into operation at the Laboratory of Acoustic Research of IKIR FEB RAS [3]. The principle of operation of a simplest interferometer is that the strainmeter base change causes additional phase increment in a laser radiation wave. The method of measurement is the following. Displacement of interferometer mirrors arranged at the base ends $l$ by the value $\lambda / 2$ results in the change of fringe pattern by one band where $\lambda$ is the light wave length on which an interferometer is operating. The total relative displacement is $\Delta l=N(\lambda / 2)$, where $N$ is the fringe pattern band number. The capabilities of the interference methods are limited by the measurement accuracy of band fraction displacement $\Delta N$,

\footnotetext{
${ }^{\star}$ Corresponding author: igor@ikir.ru
} 
which is determined by fringe pattern sharpness $F_{k}$ and is characterized by the relation $F_{k}=\frac{\Delta \lambda}{\delta \lambda}$, i.e. it is a relation of the distance between the maxima to the maximum half width $\delta \lambda$.

The advantage of a laser strainmeter against the mechanical analogues is the absence of a mechanical sensitive element. Limitations on the registration accuracy are imposed on the measurements, carried out by an "open" strainmeter without a light guide. They are determined by the effect of meteorological parameter variations. The strongest effects are those from wind, precipitations, air temperature and pressure. To exclude the wind and precipitation effects, special cover is used. Air pressure and temperature changes are quite slow processes which occur within several hours that allows us to estimate faster deformation processes. On the basis of estimation data, the strainmeter, installed in such conditions, has the accuracy of relative deformation measurement not less than $10^{-8}$ $[4,5]$.

A differential microbarometer ISGM-03M (Fig. 1), produced by «Geofizichesie izmereniya» scientific-engineering center (Novosibirsk), was used to measure the atmospheric acoustic radiation. It is installed 4 meters from the strainmeter in a pit at the depth of $0.5 \mathrm{~m}$ from the ground surface and covered from precipitation. The microbarometer is intended for the transformation of atmospheric pressure variations into the electric channel in the operating frequency band from 0.02 to $4 \mathrm{~Hz}$ and in the amplitude range from 0.001 to $100 \mathrm{~Pa}$.

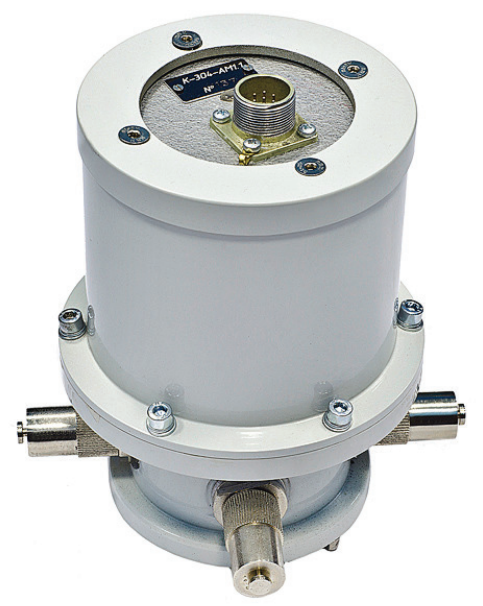

Fig. 1. Overall view of the microbarometer ISGM-03M

The principle of the device operation is based on the measurement of elastic membrane displacement relatively a fixed electrode under the effect of atmospheric pressure by a capacitance sensor of displacement. The capacitive transducer of membrane displacement is powered from a reference frequency generator stabilized in amplitude and frequency to decrease instrumentation amplifier internal self-noise. To decrease the zero drift and the amplifier low-frequency in the operating temperature range radically, a signal isolating transformer was introduced into the scheme. The primary coil is also used as high frequency filter (HFF) element. When an amplifier is constructed in this way, zero temperature drift is produced by the final stage, having a minimum amplification factor, and does not exceed $0.1 \mathrm{mkV}$ per Celsius degree. Thus, the amplifier self-noise level is determined by input stage noise on the reference frequency and reduced to the minimum by selection of corresponding elements. 
The membrane assemblies of the microbarometer, including a membrane, backplate (the base of which is fused quartz), a tube for static pressure balancing and connected with the environment air via four acoustic inputs, provide self-noise level of the microbarometer and its high temperature stability as well as small size and low weight. A thin membrane made of a corrosion-resistant alloy with controllable tension is used in the membrane unit construction. Membrane tension force stability in operating temperature range is achieved by a temperature-compensated construction of the membrane unit that provides the conversion factor (sensitivity) consistency of the microbarometer and additional decrease of self-noise.

The important feature of the experiment is the fact that the devices, installed close to each other, are covered by a three-meter snow layer in winter-spring season that provides temperature stabilization. It is controlled by a temperature sensor mounted at the basis of the strainmeter optical system. To analyze the effect of other meteorological factors, we applied the data from a weather station located 20 meters from the strainmeter. We should note that atmospheric pressure changes slowly within a day and does not affect fast deformation processes and the microbarometer owing to the tube for static pressure relief. Wind velocity has a significant impact on the instrument readings which manifests as increases in signal amplitude oscillations.

\section{Main results and discussion}

The rates of deformation and acoustic pressure changes were under analysis. A method described in the papers [3,4,5] was used. Fig. 2 shows the graphs of deformation (Fig. $2 a$ ) and acoustic pressure rates (Fig. 2b) compared with wind velocity (Fig. 2c). It is clear from the graphs that wind puts variable pressure on the snow cover and thus affects the instrument readings in the form of increased rate of observable value changes.

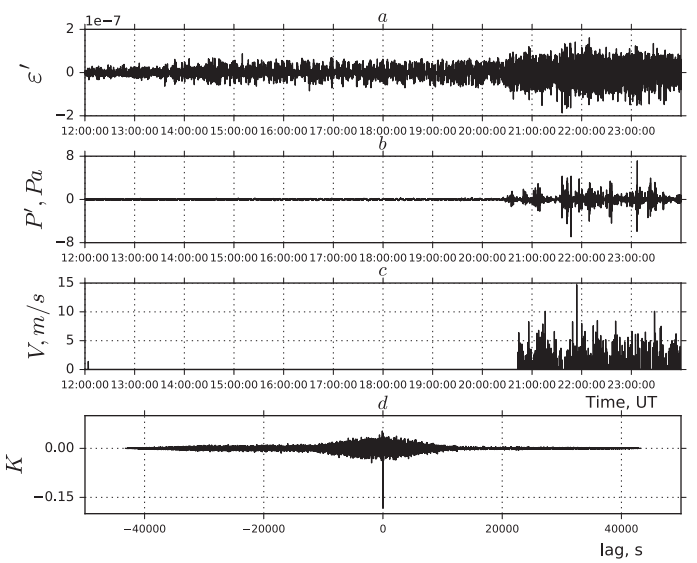

26.02.2017

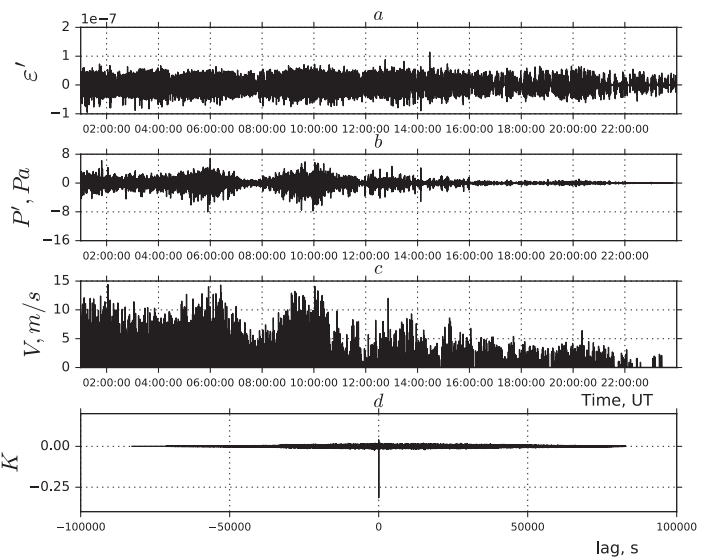

18.04.2017

Fig. 2. Fragment of acoustic-deformation change record during strong wind effect: $a$ - deformation change rate, $b$ - pressure variation change rate, $c$ - wind velocity, $d$ - cross-correlation function of deformation and pressure rate series

To estimate the correlation of strainmeter and the microbarometer data, we constructed a crosscorrelation function between the value series under investigation (Fig. 2(d)). It is clear that the 
correlation coefficient is small $(K<-0.15)$, but the peak at the zero log with negative sign is clearly defined.

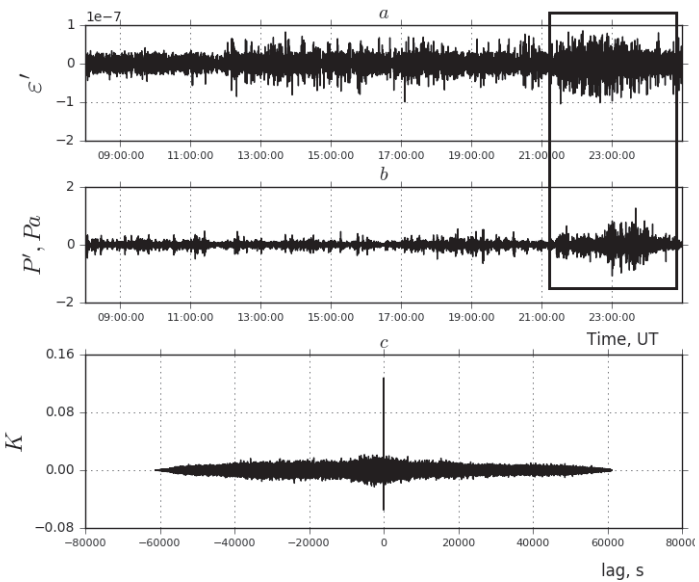

26.03.2017

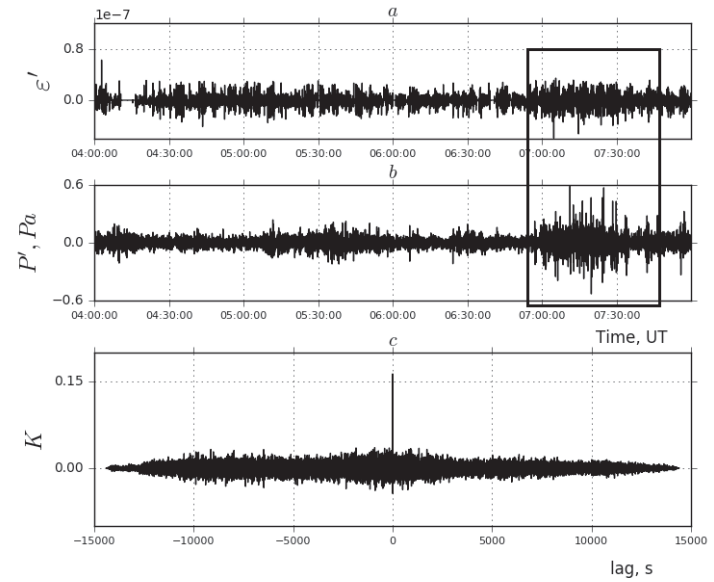

29.03.2017

Fig. 3. Fragment of acoustic-deformation change record at the absence of wind effect: $a$ - deformation change rate, $b$ - pressure variation change rate, $c$ - cross-correlation function of deformation and pressure rate series

We also chose the periods of absence of wind effect during the period under observations, they are shown in Fig. 3. It is clear from the presented fragments of estimation of deformation (Fig. $3 a$ ) and acoustic pressure (Fig. 3b) rates during the wind absence that their simultaneous increases are sometimes observed. The most vivid segments are marked by rectangles in Fig. 3. Thus, we may suppose that the occurring deformation disturbances cause acoustic vibration not only in solid medium [3-5] but also during the solid-gas transition.

For the data series in Fig. 3(a-b), cross-correlation function is also calculated (Fig. $3 c$ ). A peak at the zero log with a positive sign is clear on the graph. Thus, in case of wind effect, a reverse correlation is registered. It may be determined by the influence on the observation system from above. At the absence of wind, simultaneous increases of rates may be determined by deformation process dynamics in the lithosphere, i.e. the influence from below that causes positive correlation. It requires further investigations.

\section{Conclusions}

In the result of joint atmospheric-lithospheric observations it was shown that increase of deformation process rate causes the increase of acoustic pressure variations in the near ground layer of the atmosphere. Taking into account the fact that acoustic wave of infrared range can propagate at long distances, they can reach the ionospheric heights in favorable conditions. Thus, the possibility of propagation of deformation acoustic waves at high altitudes in the atmosphere is shown.

Differently directed effect of disturbing factors on strainmeter and microbarometer readings cause different phase of observable value rate change. 


\section{References}

[1] V.V. Adushkin, A.A. Spivak , S.B. Kishkina et al., Izvestiya Physics of the Solid Earth, 7, 34-51 (2006)

[2] V.V. Kuznetsov, V.V. Plotkin, S.Yu. Khomutov, Doklady Earth Sciences,370, 243-248 (2000).

[3] I.A. Larionov, Y.A. Nepomnyashchiy, E3S Web of Conferences, 11, 00010 (2016)

[4] I.A. Larionov, Y.V. Marapulets, and B.M. Shevtsov, Solid Earth, 5, 1293-1300 (2014)

[5] Yu.V. Marapulets, B.M. Shevtsov, I.A. Larionov, M.A. Mishchenko, A.O. Shcherbina, and A.A. Solodchuk, Russian Journal of Pacific Geology, 6, 457-464 (2012) 